\title{
The trivacancy and trivacancy-oxygen family of defects in silicon
}

\author{
V.P. Markevich ${ }^{1, a}$, A.R. Peaker ${ }^{1, b}$, B. Hamilton ${ }^{1, c}$, S.B. Lastovskii ${ }^{2, d}$, \\ L.I. Murin ${ }^{2, e}$, J. Coutinho ${ }^{3, f}$, M.J. Rayson ${ }^{4, g}$, P.R. Briddon ${ }^{5, e}$, B.G. Svensson ${ }^{6, h}$
}

${ }^{1}$ Photon Science Institute, University of Manchester, Manchester M13 9PL, United Kingdom

${ }^{2}$ State Scientific and Production Association "Scientific-Practical Materials Research Centre of the National Academy of Sciences of Belarus", Minsk 220072, Belarus

${ }^{3}$ I3N, Department of Physics, University of Aveiro, 3810-193 Aveiro, Portugal

${ }^{4}$ Department of Engineering Sciences and Mathematics, Luleå University of Technology, Luleå S-97187, Sweden

\author{
${ }^{5}$ School of Electrical, Electronic and Computer Engineering, Newcastle University, \\ Newcastle upon Tyne NE1 7RU, United Kingdom \\ ${ }^{6}$ Department of Physics, Oslo University, N-0316 Oslo, Norway \\ av.markevich@manchester.ac.uk, ${ }^{\mathrm{b}}$ peaker@manchester.ac.uk, \\ cbruce.hamilton@manchester.ac.uk, lastov@ifttp.bas-net.by, ${ }^{\mathrm{e}}$ murin@ifttp.bas-net.by \\ fjose.coutinho@ua.pt, ${ }^{9}$ mark.rayson@ltu.se, ${ }^{e}$ patrick.briddon@ncl.ac.uk, \\ hb.g.svensson@fys.uio.no
}

Keywords: silicon, trivacancy, trivacancy-oxygen, energy levels, ab-initio modeling.

\begin{abstract}
The data obtained recently from combined deep-level-transient spectroscopy (DLTS), local vibrational mode (LVM) spectroscopy and ab-initio modeling studies on structure, electronic properties, local vibrational modes, reconfiguration and diffusion paths and barriers for trivacancy $\left(\mathrm{V}_{3}\right)$ and trivacancy-oxygen $\left(\mathrm{V}_{3} \mathrm{O}\right)$ defects in silicon are summarized. New experimental results on the introduction rates of the divacancy $\left(\mathrm{V}_{2}\right)$ and trivacancy upon $4 \mathrm{MeV}$ electron irradiation and on the transformation of $\mathrm{V}_{3}$ from the fourfold coordinated configuration to the (110) planar one upon minority carrier injection are reported. Possible mechanisms of the transformation are considered and discussed.
\end{abstract}

\section{Introduction}

The trivacancy is one of the most abundant defects in Si irradiated with high-energy particles (ions, neutrons or electrons with $E>5 \mathrm{MeV}$ ) [1,2]. The $\mathrm{V}_{3}$ defect is responsible for the degradation of silicon detectors and bipolar transistors upon irradiations with high energy particles [1,3,4], so, understanding details of its electronic structure and dynamic properties is needed for developing methods of defect engineering for improving the radiation tolerance of the Si-based devices. Despite the many reports for several decades that call for its existence, the structure, electronic levels and dynamic characteristics of the $\mathrm{V}_{3}$ defect have not been identified unambiguously until recently.

We have shown in recent combined deep level transient spectroscopy (DLTS) and density functional theory (DFT) modeling studies $[2,5,6]$ that the trivacancy in the neutral charge state in Si has a fourfold coordinated (FFC) configuration with trigonal $\left(D_{3}\right)$ symmetry. The $\mathrm{V}_{3}$ defect in the FFC configuration has lower formation energy than in the (110) planar structure, which for many years was thought to be the lowest energy configuration [7,8]. The $\mathrm{V}_{3}(110)$ planar structure has $C_{2 v}$ symmetry and is sometimes referred to as part of the hexagonal ring (PHR) configuration. The FFC configuration of the silicon trivacancy was first predicted in a DFT study by Makhov and Lewis [9]. It consists of three interstitial $\mathrm{Si}$ atoms passivating three groups of four dangling bonds in a hexavacancy [9]. The PHR and FFC configurations of $\mathrm{V}_{3}$ in Si can be seen in Figs. 1(a) and 1(b), respectively. The PHR configuration was found to be the lowest energy structure for the charged $V_{3}$ defect $[2,5,6]$. Such a configuration gives rise to two acceptor levels at 0.36 and $0.46 \mathrm{eV}$ below the 


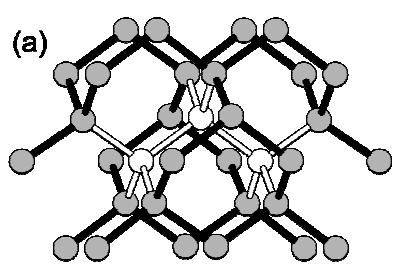

(b)
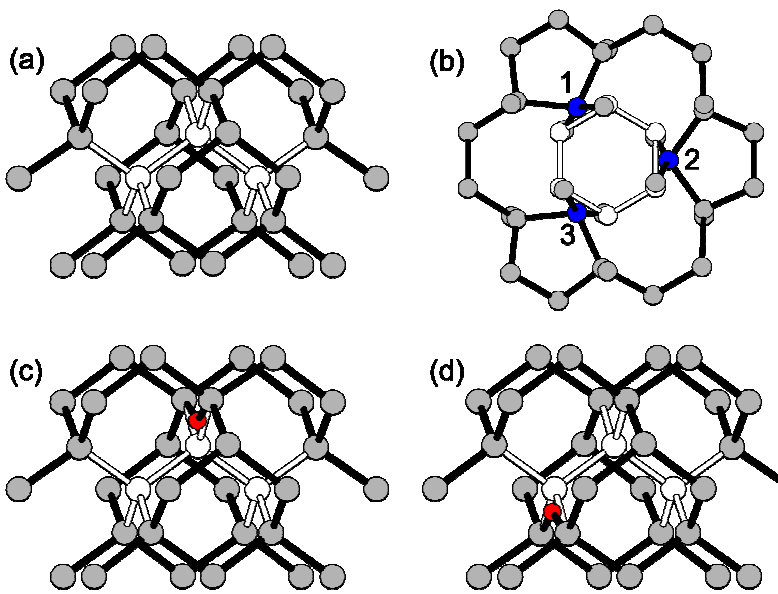

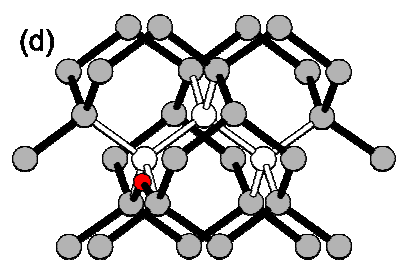

Fig. 1. Atomic structure of (a) $\mathrm{V}_{3}(\mathrm{PHR})$, (b) $\mathrm{V}_{3}(\mathrm{FFC})$, (c) $\mathrm{V}_{3} \mathrm{O}\left(\mathrm{C}_{2 \mathrm{v}}\right)$ and (d) $\mathrm{V}_{3} \mathrm{O}\left(\mathrm{C}_{1 \mathrm{~h}}\right)$. $\mathrm{V}_{3}$ (FFC) is represented along the $\langle 111\rangle$ direction, whereas other structures are viewed approximately along $\langle 110\rangle$. Silicon, oxygen and vacancy sites are represented as gray, red (twofold coordinated) and white balls, respectively. Three Si interstitial atoms in (b) are represented in blue and labeled as 1,2 and 3 .

conduction band edge $\left(E_{\mathrm{c}}\right)$ and to two donor levels at 0.19 and $0.11 \mathrm{eV}$ above the valence band edge $\left(E_{\mathrm{v}}\right)$, while in the FFC configuration it only has an acceptor level at $E_{\mathrm{c}}-0.075 \mathrm{eV}$. Five signals observed in the DLTS spectra of electron-irradiated Si diodes were associated with charge carrier emission from the above levels $[2,5,6,10]$. Fig. 2 shows the DLTS spectra with the $\mathrm{V}_{3}$-related signals indicated. It should be mentioned that immediately after electron irradiations, only DLTS signals due to the $\mathrm{V}_{3}$ defect in the PHR configuration were detected. However, storage for a few weeks of the irradiated $\mathrm{Si}$ samples at room temperature or shorter anneals in the temperature range $50-100{ }^{\circ} \mathrm{C}$ resulted in the transformation of $\mathrm{V}_{3}$ from the PHR configuration into the most stable $\mathrm{V}_{3}$ (FFC). The defect could be restored to the metastable PHR configuration by application of forward bias injection with a current density in the range $10-15 \mathrm{~A} / \mathrm{cm}^{2}$ for $10-20$ $\min$ at about $300 \mathrm{~K}$ (Fig. 2) $[2,3,5,6]$. The above transformations between the FFC and PHR configurations were found to be fully reversible in both the electron-irradiated $n$ - and $p$-type $\mathrm{Si}$ samples $[2,5,6]$. We have studied the transformation kinetics of $\mathrm{V}_{3}$ from the metastable PHR configuration (being in either neutral or singly negatively charged state) to the stable FFC one. The energy barrier for the transformation was found to be about $1.15 \mathrm{eV}$ for both the $\mathrm{V}_{3}{ }^{0}(\mathrm{PHR})$ and $\mathrm{V}_{3}{ }^{-}$(PHR) defects [10]. From DFT modeling the transformation paths for the process in both charged states were determined [10].
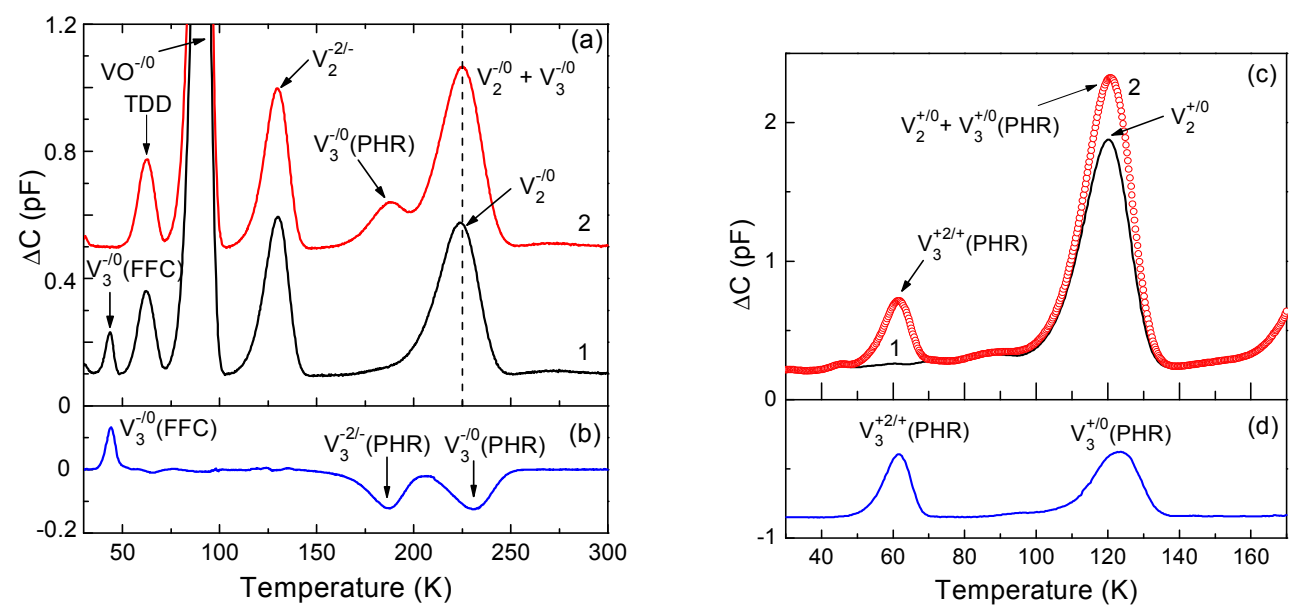

Fig. 2. DLTS spectra for a) $\mathrm{p}^{+}-\mathrm{n}-\mathrm{n}^{+}$and c) $\mathrm{n}^{+}-\mathrm{p}-\mathrm{p}^{+}$diodes, which were irradiated with $6 \mathrm{MeV}$ to doses of $8 \times 10^{13} \mathrm{~cm}^{-2}$ and $8 \times 10^{14} \mathrm{~cm}^{-2}$, respectively. The spectra a)-1 and c)-1 were recorded after annealing of the irradiated diodes at $100{ }^{\circ} \mathrm{C}$ for $30 \mathrm{~min}$. The spectra a) -2 and c)-2 were recorded after forward bias injection with a current density $10 \mathrm{~A} / \mathrm{cm}^{2}$ for $10 \mathrm{~min}$ at $300 \mathrm{~K}$ after the heattreatments at $100{ }^{\circ} \mathrm{C}$. Measurement settings for all the spectra were: $e_{\mathrm{n}}=80 \mathrm{~s}^{-1}$, bias $-10 \mathrm{~V} \rightarrow-2 \mathrm{~V}$, pulse length $1 \mathrm{~ms}$. Figs. 2b) and 2d) show differences between the spectra 2 and 1 in Figs. 2a) and $2 c)$. 
It was concluded from annealing experiments that $\mathrm{V}_{3}$ is mobile in silicon at temperatures higher than $200{ }^{\circ} \mathrm{C}$ and in oxygen-rich Si crystals it can be trapped by an oxygen atom so resulting in the appearance of a $\mathrm{V}_{3} \mathrm{O}$ defect [2,6,11-14]. The $\mathrm{V}_{3} \rightarrow \mathrm{V}_{3} \mathrm{O}$ transformation kinetics was studied in detail and the energy barrier for diffusion of the trivacancy in the neutral charge state was derived as $1.47 \mathrm{eV}[11,12]$. Two acceptor levels at 0.34 and $0.455 \mathrm{eV}$ below $E_{\mathrm{c}}$ and to two donor levels at 0.23 and $0.12 \mathrm{eV}$ above $E_{\mathrm{v}}$ were assigned to the $\mathrm{V}_{3} \mathrm{O}$ complex [2,6]. In an infrared absorption study a line at $840 \mathrm{~cm}^{-1}$ was argued to be related to a LVM of the $\mathrm{V}_{3} \mathrm{O}$ defect $[13,14]$. Regarding the possible structures of the $\mathrm{V}_{3} \mathrm{O}$ defect, in early electron spin resonance (ESR) studies of neutron-irradiated $\mathrm{Si}$ the Si-P4 and Si-P5 signals were attributed to two different configurations of the $\mathrm{V}_{3} \mathrm{O}$ defect [15]. The configuration of the defect responsible for the Si-P5 signal was found to be compatible with $C_{2 v}$ symmetry while the symmetry of the defect giving rise to the Si-P4 signal was determined to be lower than $C_{2 v}$ [15].

The results of our ab-initio modeling studies showed that the $\mathrm{O}$ atom stabilizes the planar structure and the $\mathrm{V}_{3} \mathrm{O}$ complex with $C_{2 \mathrm{v}}$ symmetry shown in Fig. 1(c) is the ground state for neutral, positively, and negatively charged defects $[2,6]$. Here, the $\mathrm{O}$ atom bridges the $\mathrm{Si}-\mathrm{Si}$ reconstruction at the center of the defect. Neutral defects were found to be energetically favorable in the spin-1 state for both $\mathrm{V}_{3} \mathrm{O}\left(C_{2 v}\right)$ and $\mathrm{V}_{3} \mathrm{O}\left(C_{1 h}\right)$. The latter defect is metastable by $0.36 \mathrm{eV}$ and it is depicted in Fig. 1(d). A fourfold coordinated $\mathrm{V}_{3} \mathrm{O}$ complex resulting from binding an oxygen atom to a $\mathrm{V}_{3}$ (FFC) structure was shown to be metastable by at least $0.2 \mathrm{eV}$ [2]. The calculated energy levels for the $\mathrm{V}_{3} \mathrm{O}\left(C_{2 v}\right)$ were close to those determined experimentally, so providing a further evidence that this configuration is the dominant one in electron-irradiated and annealed $\mathrm{Cz}$-Si crystals [2].

The results obtained on the $\mathrm{V}_{3}$ and $\mathrm{V}_{3} \mathrm{O}$ defects in silicon, which are briefly described above, have provided a solid basis for the identification of these defects, which is nearly complete. It seems that the most intriguing question still to be answered is related to revealing the $V_{3}(F F C) \rightarrow$ $\mathrm{V}_{3}$ (PHR) transformation mechanism upon injection of minority carriers. This phenomenon has been found to occur upon forward-bias-induced minority carrier injection into electron- and neutron irradiated Si diodes $[2-4,6]$ but a detailed study of the process has not been carried out yet.

In the present work new experimental results are reported on the introduction rate of trivacancy in $\mathrm{Si}$ upon irradiation with $4 \mathrm{MeV}$ electrons and on the transformation of $\mathrm{V}_{3}$ from the fourfold coordinated configuration to the (110) planar one in irradiated Si diodes upon forward-current induced minority carrier injection. Possible mechanisms of the $V_{3}(F F C) \rightarrow V_{3}$ (PHR) transformation are considered and discussed.

\section{Experimental methods and sample preparation}

Experimental results in the present work were obtained from DLTS and high-resolution Laplace DLTS (L-DLTS) [16] measurements on electron-irradiated $\mathrm{p}^{+}-n-n^{+}$and $n^{+}-p-p^{+}$diodes. $\mathrm{P}^{+}-n-n^{+}$ diodes were formed by implantation and subsequent thermal activation of boron ions into phosphorus-doped $(\rho \approx 30 \Omega \cdot \mathrm{cm}$ ) epi-Si grown on highly Sb-doped bulk Czochralski-grown $(\mathrm{Cz}) \mathrm{Si}$ wafers. Two sets of $\mathrm{n}^{+}-\mathrm{p}-\mathrm{p}^{+}$diodes were prepared. One set of the diodes was produced on borondoped epi-Si $(\rho \approx 20 \Omega \cdot \mathrm{cm}$ ), which was grown on highly boron-doped $(\rho \approx 0.005 \Omega \cdot \mathrm{cm}) \mathrm{Cz}-\mathrm{Si}$ wafers. The diodes were formed by implantation of phosphorus ions with subsequent annealing at $1150{ }^{\circ} \mathrm{C}$ in nitrogen-oxygen gas ambient. $\mathrm{N}^{+}-\mathrm{p}-\mathrm{p}^{+}$diodes from another set were prepared by phosphorus diffusion at about $1000{ }^{\circ} \mathrm{C}$ from $\mathrm{PCl}_{3}$ gas ambient into a boron-doped $(\rho \approx 5 \Omega \cdot \mathrm{cm}) \mathrm{Cz}$ Si wafer with an oxygen content of about $7.5 \times 10^{17} \mathrm{~cm}^{-3}$. The back side of the wafer was boron implanted followed by laser annealing in order to create a $\mathrm{p}^{+}$layer for contacting. Oxygen concentration in the epi-layers was estimated from the rate of transformation of the divacancy $\left(\mathrm{V}_{2}\right)$ to the divacancy-oxygen defect with the use of data presented in Ref. [17]. The oxygen concentration was (3-4) $\times 10^{17} \mathrm{~cm}^{-3}$ in all the epi-Si samples.

All the samples were irradiated with either 4 or $6 \mathrm{MeV}$ electrons at room temperature with the use of a linear accelerator. The flux of electrons was $1 \times 10^{12} \mathrm{~cm}^{-2} \mathrm{~s}^{-1}$. 
Forward-bias-injection and thermal-annealing treatments of the irradiated structures at temperatures lower than $400 \mathrm{~K}$ were carried in a He-closed-cycle cryostat in vacuum.

Concentrations of trivacancy in the $\mathrm{V}_{3}(\mathrm{PHR})$ and $\mathrm{V}_{3}$ (FFC) configurations in $\mathrm{p}^{+}-\mathrm{n}^{-} \mathrm{n}^{+}$structures were determined from magnitudes of capacitance changes due to $\mathrm{V}_{3}\{\mathrm{PHR}(2-/-)\}$ and $\mathrm{V}_{3}\{\mathrm{FFC}(-/ 0)\}$ transitions measured with L-DLTS in the temperature ranges $175-185 \mathrm{~K}$ and 40-50 K, respectively. Concentration of trivacancy in $\mathrm{V}_{3}(\mathrm{PHR})$ configuration in $\mathrm{n}^{+}-\mathrm{p}-\mathrm{p}^{+}$structures was determined from magnitudes of capacitance changes due to $\mathrm{V}_{3}\{\mathrm{PHR}(2+/+)\}$ transition measured with L-DLTS in the temperature range $60-70 \mathrm{~K}$.

\section{Ab-initio modeling details}

Electronic structure calculations were carried out using a density-functional code (AIMPRO) [18], along with the local spin density approximation (LSDA) for the exchange correlation potential [19]. Si $1 s, 2 s$ and $2 p$ core states were accounted for by fully separable dual-space pseudopotentials [20], whereas valence states, i.e. Kohn-Sham eigenstates, were expressed as linear combinations of Cartesian-Gaussian atom centered functions with angular momentum up to $l=2$. Accordingly, all Si atoms were assigned with $8 s p$ basis functions plus $5 d$-type polarization functions. Potential terms and charge density were Fourier transformed using a plane-wave basis set with an energy cut-off set at $E_{\text {cut }}=80$ Ry.

The host crystal was accounted for by using 512-atom cubic supercells, from which three atoms were removed to produce a trivacancy center. All atoms are allowed to move along their forces during a conjugate gradient atomic relaxation cycle to minimize the energy. This was carried out until total energy and atom position changes dropped below $0.3 \mathrm{meV}$ and $5 \times 10^{-5} \AA$, respectively. The Brillouin zone was sampled at $\mathbf{k}=\Gamma$, and under these conditions, the equilibrium lattice parameter and bulk modulus were $a_{0}=5.3947 \AA$ and $\mathrm{B}=98.3 \mathrm{GPa}$, whereas the indirect KohnSham energy gap and total energy gap were $\varepsilon_{\mathrm{g}}=0.56 \mathrm{eV}$ and $E_{\mathrm{g}}=A_{\mathrm{e}}-I_{\mathrm{e}}=1.33 \mathrm{eV}$. Here $A_{\mathrm{e}}$ and $I_{\mathrm{e}}$ stand for the electron affinity and ionization potential, respectively, calculated from 512-atom bulk charged supercells (compensated by an uniform background charge of opposite sign).

Migration/transformation paths were investigated with help of an improved Nudged Elastic Band (NEB) method [21]. As a starting point, a series of intermediate structures were linearly interpolated between the initial and final configurations, with each pair of consecutive structures being coupled by a virtual elastic band. While the elastic band introduces a penalty for too small or too large distances between neighboring structures, it brings them into contact with the minimum energy path connecting initial and final states (see for instance Refs. [22] and [23]).

\section{Results and discussion}

Introduction rates of the $V_{2}$ and $V_{3}$ defects in Si upon irradiation with $4 \mathrm{MeV}$ electrons. The available information about introduction rates of the $V_{3}$ defect upon irradiation of Si crystals with different high energy species is very limited. It was mentioned that the concentration of trivacancies, $\left[\mathrm{V}_{3}\right]$, was about $20-25 \%$ of the $\mathrm{V}_{2}$ concentration, $\left[\mathrm{V}_{2}\right]$, in $n$ - and $p$-type $\mathrm{Si}$ crystals irradiated with 4-6 MeV electrons at room temperature [2,6]. We have measured concentrations of the $\mathrm{V}_{2}$ and $\mathrm{V}_{3}$ defects in $n$-type epi-Si diodes irradiated with different doses of $4 \mathrm{MeV}$ electrons. The obtained dependencies are shown in Fig. 3. The concentrations of both the $V_{2}$ and $V_{3}$ defects linearly increase with the accumulated dose in the dose range studied. The values of the introduction rates were found to be about $1.25 \times 10^{-2} \mathrm{~cm}^{-1}$ and $3.0 \times 10^{-3} \mathrm{~cm}^{-1}$ for $\mathrm{V}_{2}$ and $\mathrm{V}_{3}$, respectively. So, the concentration of the $V_{3}$ defect is about $25 \%$ of the $V_{2}$ concentration for the considered case of irradiation with $4 \mathrm{MeV}$ electrons. It can be expected that the $\left[\mathrm{V}_{3}\right] /\left[\mathrm{V}_{2}\right]$ ratio will be even higher after irradiation of Si crystals with higher energy $(\mathrm{E} \geq 5 \mathrm{MeV})$ electrons, fast neutrons and heavier highenergy particles. 


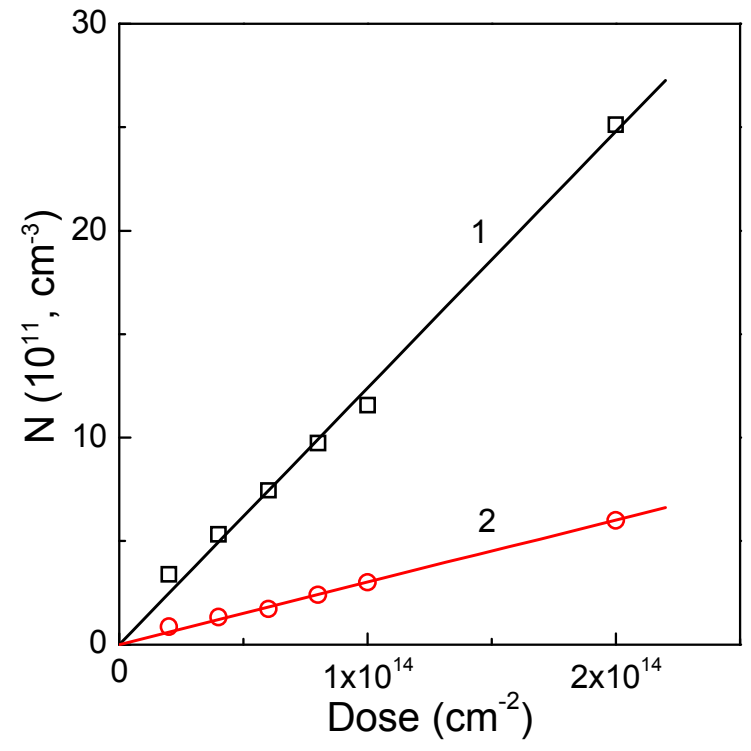

Fig. 3. Concentration of the (1) $V_{2}$ and (2) $V_{3}$ defects in $\mathrm{p}^{+}-\mathrm{n}-\mathrm{p}^{+}$epi-Si diodes versus dose of irradiation with $4 \mathrm{MeV}$ electrons. Solid lines represent the linear fits to the data obtained experimentally.
Transformation of $V_{3}$ from the FFC configuration to the (110) planar configuration upon forward-current-induced minority carrier injection: experimental results. It was found in previous studies $[2,3,5,6]$ that $\mathrm{V}_{3}$ could be transformed from the FFC configuration to the PHR one by the application of forward bias injection with a current density in the range $10-15 \mathrm{~A} / \mathrm{cm}^{2}$ for $10-20 \mathrm{~min}$ at about $300 \mathrm{~K}$. A detailed study of the $\mathrm{V}_{3}$ (FFC) $\rightarrow \mathrm{V}_{3}$ (PHR) transformation has not been carried out so far and the mechanism of this process is not understood.

Fig. 4 shows kinetics of the disappearance of the $\mathrm{V}_{3}$ defect in FFC configuration and growth of the $\mathrm{V}_{3}$ concentration in (110) planar configuration in an electron-irradiated $\mathrm{p}^{+}-\mathrm{n}-\mathrm{n}^{+}$ epi-Si diode upon the application of $1 \mathrm{~A} / \mathrm{cm}^{2}$ forward current at $325 \mathrm{~K}$. An analysis shows that both the decay of $\mathrm{V}_{3}(\mathrm{FFC})$ and the growth of $\mathrm{V}_{3}(\mathrm{PHR})$ can be described by monoexponential functions with matching decay and growth rates. It will be further shown that the rate of the $\mathrm{V}_{3}$ (FFC) $\rightarrow \mathrm{V}_{3}$ (PHR) transformation depends on the injection current $(\mathrm{J})$ and temperature, however, it has been confirmed by many experiments that the process can be described satisfactorily by a mono-exponential function at all the applied current densities and temperatures for all the $\mathrm{p}^{+}-\mathrm{n}-\mathrm{n}^{+}$and $\mathrm{n}^{+}-\mathrm{p}-\mathrm{p}^{+}$diodes studied. So, the transformation process at different conditions can be characterized by the $\mathrm{V}_{3}$ (FFC) decay or $\mathrm{V}_{3}(\mathrm{PHR})$ growth rates, which are equal and can be obtained from the analysis of the corresponding kinetics.

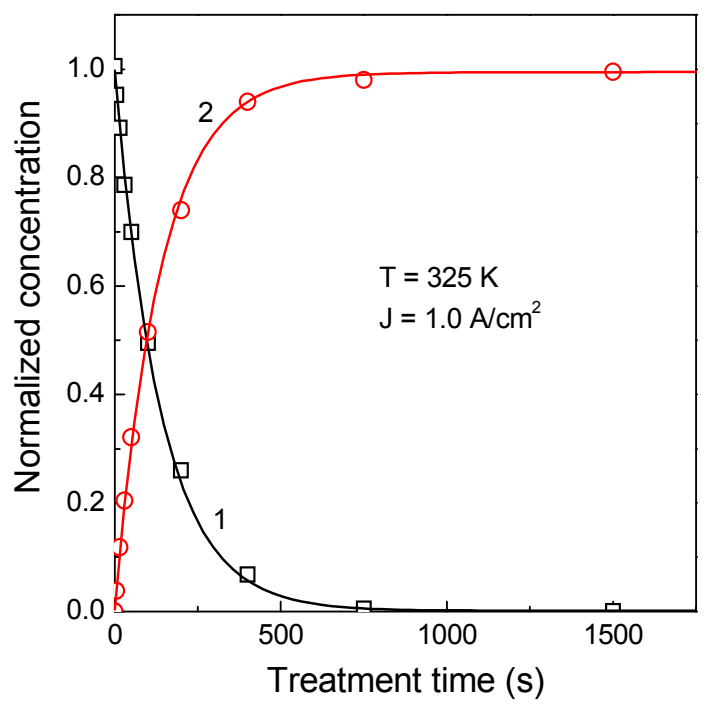

Fig. 4. Normalized concentration $\left(\mathrm{N} / \mathrm{N}_{\max }\right)$ of the (1) $\mathrm{V}_{3}$ (FFC) and (2) $\mathrm{V}_{3}$ (PHR) defects in an electron irradiated $\mathrm{p}^{+}-n-\mathrm{n}^{+}$epiSi diode versus time of exposure of the diode to $1 \mathrm{~A} / \mathrm{cm}^{2}$ forward current at $325 \mathrm{~K}$.
Fig. 5 shows temperature dependencies of the $\mathrm{V}_{3}$ (FFC) $\rightarrow \mathrm{V}_{3}$ (PHR) transformation rates in different diodes for the current densities of 0.5 and 2 $\mathrm{A} / \mathrm{cm}^{2}$. The obtained dependencies are not trivial. In the temperature range $35-150 \mathrm{~K}$ the transformation process is nearly athermal, then the transformation rates decrease significantly in all the diodes studied as the temperature rises from $150 \mathrm{~K}$ to $250 \mathrm{~K}$. Finally, the transformation rates increase in the temperature range $250-325 \mathrm{~K}$. It can be seen that the transformation rate increases with the increase in the current density in the whole temperature range studied. This is an expectable result. The transformation rates are found to be different for different samples at the same value of the forward current density. It appears that the transformation rate could be inversely proportional to the equilibrium carrier density in semiconductor base $\left([n] \approx 1 \times 10^{14} \mathrm{~cm}^{-3}\right.$ for epi $\mathrm{n}-\mathrm{Si},[p] \approx 3 \times 10^{14} \mathrm{~cm}^{-3}$ for epi p-Si, and $[p] \approx 2 \times 10^{15} \mathrm{~cm}^{-3}$ for $\mathrm{Cz} \mathrm{p}$-Si), however further studies are necessary to determine the main factor responsible for the different $\mathrm{V}_{3}(\mathrm{FFC}) \rightarrow$ $\mathrm{V}_{3}$ (PHR) transformation rates in different materials. 


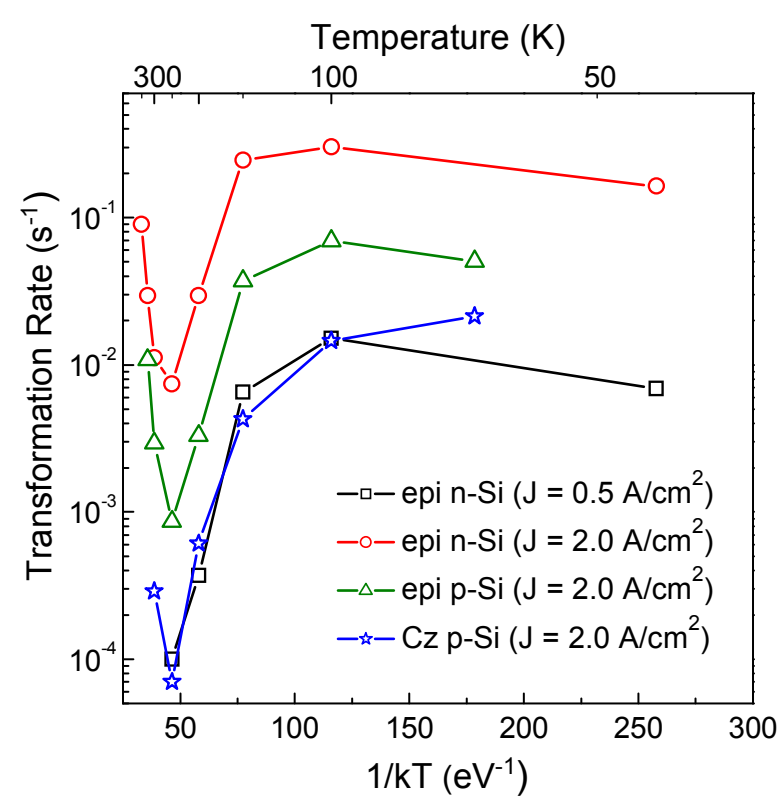

Fig. 5. Temperature dependencies of the $\mathrm{V}_{3}$ (FFC) $\rightarrow \mathrm{V}_{3}$ (PHR) transformation rates in different diodes.

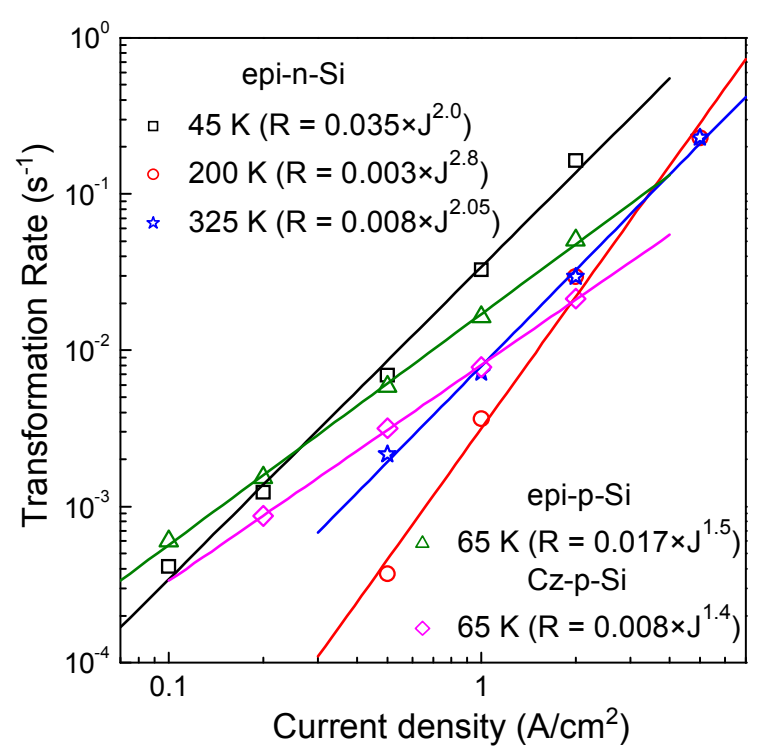

Fig. 6. Injection current dependencies of the $\mathrm{V}_{3}$ (FFC) $\rightarrow \mathrm{V}_{3}$ (PHR) transformation rates at different temperatures for different diodes.

To get further insight into the mechanisms responsible for the transformation process we have studied dependencies of the transformation rates $(\mathrm{R})$ on the injection current density at different temperatures. The results are shown in Fig. 6. The dependencies obtained can be approximated satisfactorily by a power low. The power values vary from about 1.5 to about 3 depending on temperature of the treatment and material. At lower temperatures $(\mathrm{T}<150 \mathrm{~K})$ the power value is 2 in $n$-type $\mathrm{Si}$ and about 1.5 in $p$-type Si materials. In $n$-type $\mathrm{Si}$ the power value increases up to 2.8 at $200 \mathrm{~K}$, the temperature in the range where the decrease in $\mathrm{R}$ values with increasing temperature occur. Finally, at higher temperature $(\mathrm{T}>300 \mathrm{~K})$ the power value is close to 2 . The power values derived indicate that interactions of at least two charge carriers with the $\mathrm{V}_{3}(\mathrm{FFC})$ defect are necessary to transform it to the PHR configuration [24,25].

Transformation of $V_{3}$ from the FFC configuration to the (110) planar configuration upon forward-current-induced minority carrier injection: modeling results and discussion. The experimental observations on the $\mathrm{V}_{3}(\mathrm{FFC}) \rightarrow \mathrm{V}_{3}$ (PHR) transformation is indicative of an athermal process leveraged by energy transfer from charge carriers, i.e. free electrons, holes or both to FFC trivacancies. This general mechanism was reviewed by Kimerling [24] and Lang [25], and basically, it was argued that a fraction of the energy of free electrons or holes captured at defect traps may be converted (non-radiatively) to defect vibrational modes [26]. This vibrational energy (which can match the trap depth), may be channeled to specific transformation coordinates, and by this way enhance atomic motion that was otherwise inhibited. Although in their original work the authors consider free-carrier capture only, the same arguments may be applied to enhanced transformation processes such as excitonic recombination-enhanced or carrier-assisted BourgoinCorbett mechanisms [27,28]. While many possibilities and mechanism combinations may take place, the situation is worsen by severe limitations in the theoretical method here employed, especially regarding the treatment of excited states and a proper account for electron-phonon interactions. Here we explore the possibility that the $V_{3}$ (FFC) $\rightarrow V_{3}$ (PHR) transformation could proceed through the metastable states, which were considered in Ref. [11] to explore the migration mechanism of $V_{3}$. The structures considered are shown in Fig. 7. Our approach was to calculate the MEP trajectories for several electronic states (Fig. 8), and from here to hypothesize and comment on a few mechanisms, that to our opinion deserve special attention. Before proceeding with the 

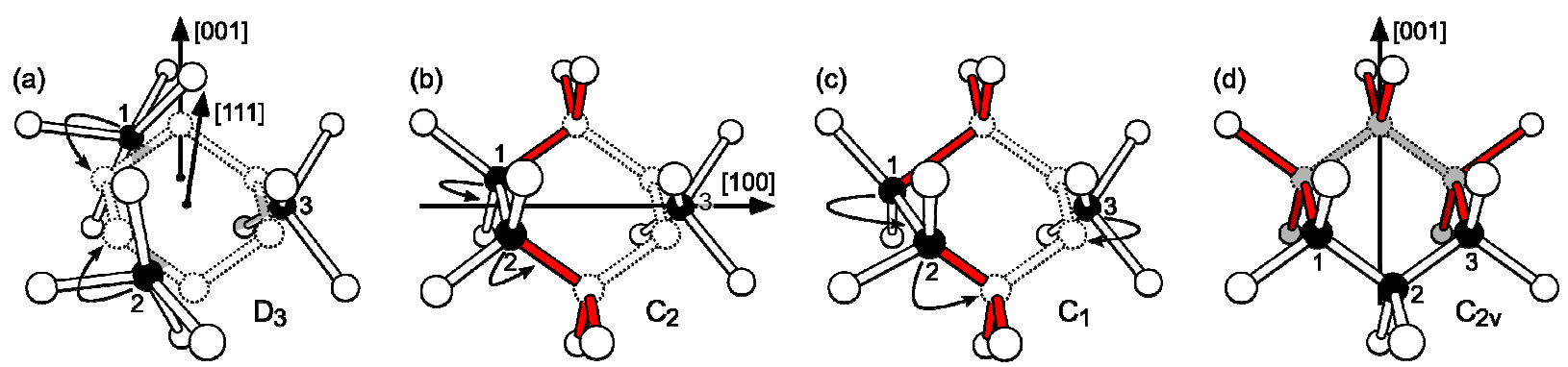

Figure 7. Atomic structure of stable and metastable $V_{3}$ complexes in silicon along with some crystallographic directions and planes. The three Si atoms 1,2 and 3 are shown in black for a better perception of their displacement with respect to the vacant lattice sites of the hexavacancy ring (drawn with a dashed line), which is clearly seen in (a). Brocken bonds are colored in red and Si atoms that are barely displaced from their lattice sites are shown in white. Structures shown in (a) and (d) are the most stable structures for the neutral and charged states, respectively, whereas metastable structures in (b) and (c) are intermediate forms along the transformation path between $\mathrm{V}_{3}(\mathrm{FFC})$ and $\mathrm{V}_{3}\left(\mathrm{C}_{2 \mathrm{v}}\right)$. Atom displacements are indicated by curved arrows.

analysis, we would like to point out that different trajectories, other than those depicted in Fig. 8, were investigated, although they had considerably higher energy barriers.

In the analysis below, energy barriers are labelled as $\Delta E^{q}(d, n)$, where $q$ stands for the charge state of the trivacancy, $d$ is the direction of the transformation along the coordinate axis in Fig. 8 (forward and reverse transformations are represented towards the right and left directions, respectively), and finally $n$ is one of the transformation coordinates $(n=1, \ldots, 4)$ depicted in Fig. 8.

(Mechanism 1). Transformation assisted by hole capture. This possibility was ruled out since there is no experimental evidence neither a theoretical prediction of a hole trap for the FFC trivacancy. Even considering the remote possibility of a shallow $(0 /+)$ trap edging the valence band

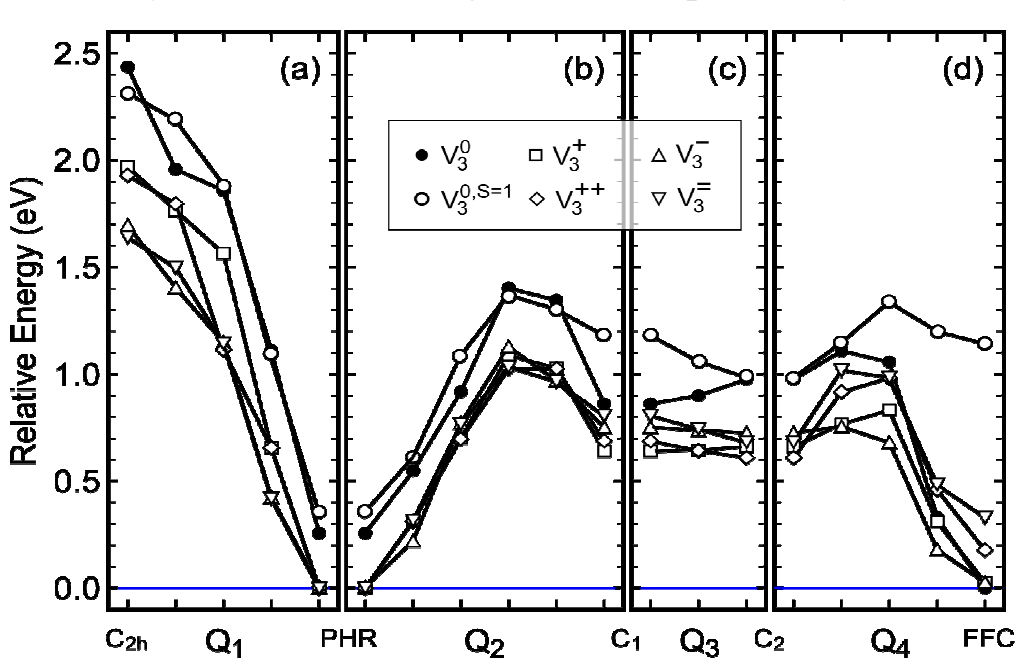

Fig. 8. Minimum energy paths obtained from CI-NEB calculations between the most relevant $\mathrm{V}_{3}$ structures and charge states for this work. Relative energies of neutral and charged defects were calculated with respect to FFC and PHR structures, respectively. Lines that connect calculated data are uniquely shown for eye guidance purposes. top, that would not provide enough energy to assist the defect to overcome the large $\Delta E^{+}(\mathrm{rev}, 4)=0.81 \mathrm{eV}$ barrier.

(Mechanism 2). Transformation assisted by electron capture. This is also unlikely as the captured energy can not be higher than $75 \mathrm{meV}$ (the depth of the acceptor level for the $\mathrm{V}_{3}$ (FFC). This is approximately one order of magnitude less than the $\Delta E^{-}(\mathrm{rev}, 4)=0.72 \mathrm{eV}$ barrier. This conclusion is also supported by the fact that the DLTS peak due to the $\mathrm{V}_{3}$ (FFC)-related $E(-/ 0)$ transition is detected at about $\mathrm{T}=$ $45 \mathrm{~K}$, implying that the electron capture energy is lower than any transformation barrier surrounding the $\mathrm{V}_{3}{ }^{-}\left(\mathrm{D}_{3}\right)$ state.

(Mechanism 3). Transformation assisted by a two-electron capture event. In analogy to Mechanism 1, this was also ruled out since there is no experimental evidence neither it is theoretically predicted that the FFC trivacancy has a second acceptor level. 
(Mechanism 4). Transformation taking place on a neutral excited state. This is similar to a carrier-assisted mechanism that may occur in bistable defects or when Jahn-Teller instabilities are rather strong [29], although in this case the capture of an exciton or two oppositely charged carriers is necessary. Typically in $\mathrm{Si}$, exciton recombination life-times on defects are as long as $\tau_{\mathrm{x}} \sim 10^{-8}$ $10^{-6} \mathrm{~s}$. This is usually a long time if we compare to typical atomic transformation time scales, $\tau_{\mathrm{t}} \sim$ $10^{-12}-10^{-13} \mathrm{~s}$, and it is possible that, upon capturing an excitation such an electron-hole pair, the FFC defect may be found now at an unstable state and quickly relax to the PHR structure. An approximation to such transformation was investigated by calculating the MEP of the trivacancy in the spin-1 state, i.e. by promoting the highest occupied spin-up electron to the next available KohnSham level. The results are shown in Fig. 8 as open circles. The transformation on this state is not athermal as we predict small $\Delta E^{0, S=1}(\mathrm{rev}, 4)=0.20 \mathrm{eV}, \Delta E^{0, S=1}(\mathrm{rev}, 3)=0.19 \mathrm{eV}$ and $\Delta E^{0, S=1}(\mathrm{rev}, 2)=$ $0.20 \mathrm{eV}$ barriers along the path. However, given the limitations of the method to treat this problem we have to consider that these could be artificially overestimated.

(Mechanism 5) Recombination enhanced transformation upon electron-hole capture. This is unlikely since the barrier to be surmounted by neutral $\mathrm{V}_{3}$ is higher than the band-gap energy.

(Mechanism 6) Recombination enhanced $\mathrm{V}_{3}^{0}\left(D_{3}\right) \rightarrow \mathrm{V}_{3}{ }^{0}\left(C_{1}\right)$ transformation, followed by carrier capture to assist the $\mathrm{V}_{3}{ }^{0}\left(C_{1}\right)+q \rightarrow \mathrm{V}_{3}{ }^{\mathrm{q}}\left(C_{2 \mathrm{v}}\right)$ step. According to the calculations, this seems to be a possible mechanism. After trapping an electron, the excess of negative charge in $\mathrm{V}_{3}{ }^{-}\left(D_{3}\right)$ can lead to hole capture, bringing the center back to the neutral state and close to $\mathrm{V}_{3}{ }^{0, S=1}\left(D_{3}\right)$. The recombination energy can be approximately $E_{\mathrm{g}}-75 \mathrm{meV} \approx 1.1 \mathrm{eV}$, which could be enough to overcome the $\Delta E^{0}(\mathrm{rev}, 4)$ barrier and reach the $\mathrm{V}_{3}{ }^{0}\left(C_{1}\right)$ metastable state. We note that the forward $\left(\mathrm{Q}_{3}, \mathrm{Q}_{4}\right)$ transformation barrier $(0.25 \mathrm{eV}$ high $)$ could be high enough to prevent a substantial recovery of $\mathrm{V}_{3}{ }^{0}\left(D_{3}\right)$ and further carriers could be captured at the $0.23 \mathrm{eV}$ and $0.30 \mathrm{eV}$ deep hole or electron traps of $\mathrm{V}_{3}\left(C_{1}\right)$ [11]. These figures are rather close to the $\Delta E^{+}(\mathrm{rev}, 2)=0.45 \mathrm{eV}$ and $\Delta E^{-}(\mathrm{rev}, 2)=0.38 \mathrm{eV}$ barriers, respectively, that the defect as to overcome before reaching the PHR structure.

\section{Summary}

Summarizing, the results available on the $\mathrm{V}_{3}$ and $\mathrm{V}_{3} \mathrm{O}$ defects in silicon allow us to state that the identification of these defects is nearly complete. Solid arguments are found showing that the trivacancy is a bistable center in the neutral charge state with a fourfold coordinated configuration being the energetically favorable one. It appears that the $V_{3}$ defect is for now the unique member from a family of mysterious "fourfold coordinated defects" [9,30] to be firmly identified.

This understanding of the electronic and dynamic properties of $\mathrm{V}_{3}$ can be used for improvement of radiation hardness of silicon detectors and bipolar transistors. It has been shown that the $\mathrm{V}_{3}$ defect in the PHR configuration is effectively introduced by irradiations with high-energy particles. In this configuration $\mathrm{V}_{3}$ has four energy levels in the gap and can significantly contribute to the degradation of silicon detectors and bipolar transistors upon irradiations. However, the PHR configuration is not the energetically favorable one and the $\mathrm{V}_{3}$ defect transforms to the FFC configuration upon storage of the irradiated devices at room temperature or shorter heat treatments in the temperature range $50-150{ }^{\circ} \mathrm{C}$. Such a transformation results in recovery of some degraded parameters of the Si devices, because in the FFC configuration the $\mathrm{V}_{3}$ defect possess only a shallow acceptor level and therefore is electrically neutral at the device operation temperatures. Obviously, short heat-treatments in the temperature range $100-150{ }^{\circ} \mathrm{C}$ can be recommended for partial recovery of the degraded Si devices.

\section{Acknowledgements}

The University of Manchester team would like to thank EPSRC-UK for financial support. J.C. thanks the NanoTP Cost Action MP0901 for funding and the FCT under Grant No. PEstC/CTM/LA0025/2011. 


\section{References}

[1] M. Ahmed, S.J. Watts, J. Matheson, and A. Holmes-Siedle, Deep-level transient spectroscopy studies of silicon detectors after $24 \mathrm{GeV}$ proton irradiation and $1 \mathrm{MeV}$ neutron irradiation, Nuclear Instruments and Methods in Physics Research A 457 (2001) 588-594.

[2] V.P. Markevich, A.R. Peaker, S.B. Lastovskii, L.I. Murin, J. Coutinho, V.J.B. Torres, P.R. Briddon, L. Dobaczewski, E.V. Monakhov, and B.G. Svensson, Trivacancy and trivacancy-oxygen complexes in silicon: Experiments and ab initio modeling, Phys. Rev. B 80 (2009) 235207-(1-7).

[3] R.M. Fleming, C.H. Seager, D.V. Lang, E. Bielejec, and J.M. Campbell, A bistable divacancylike defect in silicon damage cascades, J. Appl. Phys. 104 (2008) 083702-(1-10).

[4] A. Junkes, I. Pintilie, E. Fretwurst, and D. Eckstein, A contribution to the identification of the E5 defect level as tri-vacancy ( $\left.\mathrm{V}_{3}\right)$, Physica B 407 (2012) 3013-3015.

[5] V.P. Markevich, A.R. Peaker, S.B. Lastovskii, L.I. Murin, J. Coutinho, A.V. Markevich, V.J.B. Torres, P.R. Briddon, L. Dobaczewski, E.V. Monakhov, and B.G. Svensson, Trivacancy in silicon: A combined DLTS and ab initio modeling study, Physica B 404 (2009) 4565-4567.

[6] V.P. Markevich, A.R. Peaker, B. Hamilton, S.B. Lastovskii, L.I. Murin, J. Coutinho, V.J.B. Torres, P.R. Briddon, L. Dobaczewski, and B.G. Svensson, Structure and electronic properties of trivacancy and trivacancy-oxygen complexes in silicon, Phys. Status Solidi A 208 (2011) 568-571.

[7] Y-H. Lee, J.W. Corbett, EPR study of defects in neutron-irradiated silicon: Quenched-in alignment under $<110>$ uniaxial stress, Phys. Rev. B 9 (1974) 4351-4361.

[8] J.L. Hastings, S.K. Estreicher, and P.A. Fedders, Vacancy aggregates in silicon, Phys. Rev. B 56 (1997) 10215-10220.

[9] D.V. Makhov, L.J. Lewis, Stable fourfold configurations for small vacancy clusters in silicon from ab initio calculations, Phys. Rev. Lett. 92 (2004) 255504-(1-4).

[10] J.H. Bleka, E.V. Monakhov, B.G. Svensson, and B.S. Avset, Room-temperature annealing of vacancy-type defect in high-purity n-type Si, Phys. Ref. B 76 (2007) 233204-(1-3).

[11] J. Coutinho, V.P. Markevich, A.R. Peaker, B. Hamiton, S.B. Lastovskii, L.I. Murin, B.G. Svensson, M.J. Rayson, and P.R. Briddon, Electronic and dynamical properties of the silicon trivacancy, Phys. Rev. B 86 (2012) 174101-(1-13).

[12] V.P. Markevich, A.R. Peaker, B. Hamilton, S.B. Lastovskii, L.I. Murin, J. Coutinho, A.V. Markevich, M.J. Rayson, P.R. Briddon, and B.G. Svensson, Reconfigurations and diffusion of trivacancy in silicon, Physica B 407 (2012) 2974-2977.

[13] L.I. Murin, B.G. Svensson, J.L. Lindstrom, V.P. Markevich, and C.A. Londos, Trivacancyoxygen complex in Si: Local vibrational mode characterization, Physica B 404 (2009) 4568-4571.

[14] L.I. Murin, B.G. Svensson, J.L. Lindstrom, V.P. Markevich, and C.A. Londos, Divacancyoxygen and trivacancy-oxygen complex in silicon: Local Vibrational Mode studies, Solid State Phenomena 156-158 (2010) 129-134.

[15] K.L. Brower, Structure of multiple-vacancy (oxygen) centers in irradiated silicon, Radiation Effects 8 (1971) 213-219.

[16] L. Dobaczewski, A.R. Peaker and K. Bonde Nielsen, Laplace-transform deep-level transient spectroscopy: The technique and its applications to the study of point defects in semiconductors, J. Appl. Phys. 96 (2004) 4689-4728.

[17] M. Mikelsen, E.V. Monakhov, G. Alfieri, B.S. Avset, and B.G. Svensson, Kinetics of divacancy annealing and divacancy-oxygen formation in oxygen-enriched high-purity silicon, Phys. Rev. B 72 (2005) 195207-(1-6). 
[18] M.J. Rayson, P.R. Briddon, Rapid iterative method for electronic-structure eigenproblems using localized basis functions, Comp. Phys. Comm. 172 (2008) 128-134.

[19] J.P. Perdew, Y Wang, Accurate and simple analytic representation of the electron-gas correlation energy, Phys. Rev. B 45 (1992) 13244-13249.

[20] C. Hartwigsen, S. Goedecker, and J. Hutter, Relativistic separable dual-space Gaussian potentials from H to Rn, Phys. Rev. B 58 (1998) 3641-3662.

[21] G. Henkelman, B.P. Uberuaga, and H.Jonsson, A climibing image nudged elastic band method for finding saddle points and minimum energy paths, J. Chem. Phys. 113 (2000) 9901-9904.

[22] A. Carvalho, R. Jones, M. Sanati, S.K. Estreicher, J. Coutinho, and P.R. Briddon, Firstprinciples investigation of a bistable boron-oxygen interstitial pair in Si, Phys. Rev. B 73 (2006) 245210-(1-7).

[23] A. Carvalho, R. Jones, C. Janke, J.P. Goss, P.R. Briddon , J. Coutinho and S. Oberg, Self interstitial in germanium, Phys. Rev. Lett. 99 (2007) 175502-(1-4).

[24] L.C. Kimerling, Recombination enhanced defect reactions, Sol. St. Electronics 21 (1978) 1391-1401.

[25] D.V. Lang, Recombination-enhanced defect reactions in semiconductors, Ann. Rev. Mater. Sci. 12 (1982) 377-400.

[26] J.D. Weeks, J.C. Tully, and L.C. Kimerling, Theory of recombination-enhanced defect reactions in semiconductors, Phys. Rev. B 12 (1985) 3286-3292.

[27] J.C. Bourgoin, J.W. Corbett, A new mechanism for interstitial migration, Phys. Lett. A 38 (1972) 135-137.

[28] J.C. Bourgoin, D. Peak, and J.W. Corbett, Ionization-enhanced diffusion: ion implantation in semiconductors, J. Appl. Phys. 44(1973) 3022-3027.

[29] Y. Bar-Yam, J.D. Joannopoulos, Barrier to migration of silicon self-interstitial, Phys. Rev. Lett. 52 (1984) 1129-1132.

[30] S. Goedecker, T. Deutsch, and L. Billard, A fourfold coordinated point defect in silicon, Phys. Rev. Lett. 88 (2002) 235501-(1-4). 
Gettering and Defect Engineering in Semiconductor Technology XV

10.4028/www.scientific.net/SSP.205-206

The Trivacancy and Trivacancy-Oxygen Family of Defects in Silicon

10.4028/www.scientific.net/SSP.205-206.181 